\title{
Rotor Phases in Compound Semiconductors
}

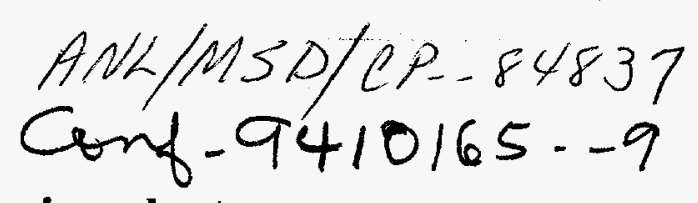

David Long Price ${ }^{1,}$ Marie-Louise Saboungi 1 , and W. Spencer Howells ${ }^{2}$

1Argonne National Laboratory

Materials Science Division

Argonne, IL 60439

2Rutherford-Appleton Laboratory

Chilton, Oxon OX11

OQX, United Kingdom

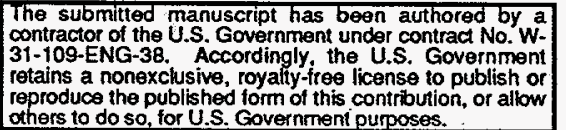

reproduce the published form of this contribution, or allow

others to do so, for U.S. Governmeni purposes.

November, 1994

\section{Distribution:}

1-2. M. J. Masek

3. B. D. Dunlap

4. G. W. Crabtree

5. J. D. Jorgensen

6. B. S. Brown

7. Editorial Office

8. Authors
This report was prepared as an account of work sponsored by an agency of the United States Government. Neither the United States Government nor any agency thereof, nor any of their employees, makes any warranty, express or implied, or assumes any legal liability or responsibility for the accuracy, completeness, or usefulness of any information, apparatus, product, or process disclosed, or represents that its use would not infringe privately owned rights. Reference herein to any specific commercial product, process, or service by trade name, trademark, manufacturer, or otherwise does not necessarily constitute or imply its endorsement, recommendation, or favoring by the United States Government or any agency thereof. The views and opinions of authors expressed herein do not necessarily state or reflect those of the United States Government or any agency thereof.

To be published in the Proceedings of the 1994 International Conference on Neutron Scattering, Sendai, Japan, October 11-14, 1994 - (Special issue of Physica B).

This work is supported by the Division of Materials Sciences, Office of Basic Energy Sciences of DOE, under contract No. W-31-109-ENG-38. 


\section{DISCLAIMER}

Portions of this document may be illegible in electronic image products. Images are produced from the best available original document. 


\title{
Rotor Phases in Compound Semiconductors
}

David Long Price ${ }^{1}$, Marie-Louise Saboungi ${ }^{1}$, and W. Spencer Howells ${ }^{2}$

\author{
1 Argonne National Laboratory \\ Materials Science Division \\ Argonne, IL 60439 \\ 2Rutherford-Appleton Laboratory \\ Chilton, Oxon OX11 \\ OQX, United Kingdom
}

/sm

Distribution:

1-2. M. J. Masek

3. B. D. Dunlap

4. G. W. Crabtree

5. J. D. Jorgensen

6. B. S. Brown

7. Editorial Office

8. Authors

\section{November, 1994}

To be published in the Proceedings of the 1994 International Conference on Neutron Scattering, Sendai, Japan, October 11-14, 1994 - (Special issue of Physica B).

This work is supported by the Division of Materials Sciences, Office of Basic Energy Sciences of DOE; under contract No. W-31-109-ENG-38. 
20 October 1994

\title{
Rotor Phases In Compound Semiconductors
}

\author{
David Long Price, ${ }^{a}$ Marie-Louise Saboungi ${ }^{a}$ and W. Spencer Howells ${ }^{b}$ \\ aArgonne National Laboratory, Argonne, IL 60439, U.S.A. \\ bRutherford-Appleton Laboratory, Chilton, Oxon OX11 0QX, U.K.
}

\begin{abstract}
Quasielastic neutron scattering is used to study the disordering processes in two classes of semiconductor: I-IV Zintl compounds and the phosphorus-selenium system. Two alkali-metalpolyvalent metal Zintl compounds, $\mathrm{CsPb}$ and $\mathrm{NaSn}$, exhibit a two-stage melting process with hightemperature solid phases characterized by rapid dynamical disorder. In $\mathrm{CsPb}$ this disorder is clearly associated with rapid reorientations of polyanions with thecations participating in the dynamical disorder on the same time scale. In $\mathrm{NaSn}$ the disorder is associated with fast reorientations of the polyanions closely coupled to a slower migration of the cations. The two high-temperature solid phases of the molecular crystal $\mathrm{P}_{4} \mathrm{Se}_{3}$ are confirmed to be rotor phases with small but significant differences in the reorientational motions in the two phases.
\end{abstract}

Keywords: Quasielastic scattering, rotor phase, semiconductor, disorder

Corresponding author:

Dr. David Long Price

Building 223, Argonne National Laboratory, Argonne.IL 60439, U.S.A.

Fax: +1-708-252-7777

E-mail: Price@anlpns.pns.anl.gov 


\section{Introduction}

In a one-component solid, melting can be thought of simply as the onset of translational disorder which breaks down the long-range order of the crystal. In a more complex system, several kinds of disorder are possible. These can include translational disorder of one sublattice (fast-ion conductors), chemical disorder between two or more sublattices (disordered alloys), orientational disorder of molecules or complex ions (rotor phases), or lattice melting in one or two directions in the crystal (ferroelastic phases). Formally, one can associate an entropy increase with each of the disordering processes involved in a particular system. Melting may occur in a form that all the disordering processes take place at one temperature - the melting point - with a characteristic entropy of melting that represents the sum of the contributions from each process. Other systems may exhibit a sequence of transitions where partial disordering takes place, each with an entropy change appropriate to the process involved; the sum of all the energy changes can be considered as the true entropy of melting. The latter systems provide an excellent viewpoint for studying the effects of the different processes involved. in melting on the physical properties of interest.

This article discusses the disordering processes in two classes of semiconductor: I-IV Zintl compounds, in which charge transfer and directional bonding lead to the formation of tetrahedral polyanions, and the phosphorus-selenium system, which forms $\mathrm{P}_{4} \mathrm{Se}_{n}$ molecular units based on the $\mathrm{P}_{4}$ tetrahedron.

\section{Zintl compounds}

Zintl compounds are formed from an electropositive metal $\mathrm{A}$ and an electronegative metal or semimetal $\mathrm{M}$; electron transfer from $\mathrm{A}$ to $\mathrm{M}$, along with directional bonding between the $\mathrm{M}^{-}$ions, 
leads to chemical behavior in these ions characteristic of elements to the right of $\mathrm{M}$ in the periodic table. This article discusses dynamic disorder in two equiatomic $\mathrm{Zintl}$ compounds, $\mathrm{CsPb}$ and $\mathrm{NaSn}$, which share a number of unusual properties with other Zintl compounds of $\mathrm{Pb}$ and $\mathrm{Sn}$, arising from the formation of tetrahedral $\mathrm{Pb}_{4}{ }^{4-}$ or $\mathrm{Sn}_{4}{ }^{4-} \mathrm{Zintl}$ ions. Crystal structures of these compounds have polyanions of this type arranged on a bct lattice, separated by alkali metal ions, each of which is shared by two polyanions [1]. In the neutron diffraction patterns of the liquids, strong "first sharp diffraction peaks" are observed, due to dense random packing of the polyanions $[2,3]$.

The enthalpy function [4] of $\mathrm{CsPb}$ is shown in Fig. 1 along with that of $\mathrm{KPb}$, to illustrate differences in melting behavior as discussed above. It is seen that, whereas KPb has a single melting transition, $\mathrm{CsPb}$ has two transitions about $50 \mathrm{~K}$ apart, with the enthalpy and entropy of melting split rather evenly between them. The structure of the three phases was established in neutron diffraction measurements [2]. The structure factors of the low-temperature ( $\beta$ ) crystalline. phase and the liquid were similar to those of other $\mathrm{Pb}$ [2]and $\mathrm{Sn}$ [3] Zintl compounds, but that of the intermediate $(\alpha)$ phase was unusual, combining a feature at $Q \sim 1 \AA^{-1}$ that appeared to be a set of unresolved Bragg peaks with a diffuse, noncrystalline pattern at larger $Q$.

To investigate the nature of the disorder, inelastic neutron scattering measurements were carried out on the IN-6 spectrometer at ILL [5]. As expected, in the quasielastic region the spectra in the $\beta$ phase had the shape of the unbroadened resolution function of the instrument, while those of the liquid had the usual lorenztian shape. The spectra of the $\alpha$ phase showed a superposition of unbroadened and broadened components, which could be well represented by a delta function and single lorentzian function, respectively, broadened by the resolution function. The intensities of . the two components as a function of $Q$ are reproduced here in Fig. 2. The delta-function intensity. is seen to peak in the region of the Bragg peaks at $Q \sim 1 \AA^{-1}$, but have a significant, non-zero value over the rest of the measured Q range, while the lorentzian-function has a small peak just to the left 
of the Bragg peaks $\left(Q \sim 0.9 \AA^{-1}\right)$ and then starts rise to large values at $Q \sim 1.5 \AA^{-1}$; the sum of the two appears to account for the static structure factor measured in the diffraction experiment [2], so there is no significant additional component to the scattering in this $Q$ range. This behavior is immediately suggestive of dynamic orientational disorder of the type found in plastic crystals and other rotor phases.

To extract quantitative information, a simple model was adopted in which the atoms were assumed to form $\mathrm{Cs}_{4} \mathrm{~Pb}_{4}$ units; these were assumed to undergo independent, random jumps between the four orientations found in the $\beta$ crystal structure in succession as one moves up the $c$ axis [5]. Since these orientations are unequally spaced, two jump times, $\tau_{1}$ and $\tau_{2}$, were assumed for nearest- and next-nearest-neighbor jumps, respectively. The quasielastic scattering spectra calculated from this model showed both unbroadened and broadened components similar to the measured ones. The integrated intensities of the two components, which are independent of the values of $\tau_{1}$ and $\tau_{2}$, are compared in Fig. 2 with the measured values, averaged over the different runs, and seen to be in excellent agreement. Models with only independent $\mathrm{Pb}_{4}{ }^{4-}$ polyanions reorienting do not reproduce the first peak in the intensity of the lorentzian component at $Q=0.9$. $\AA^{-1}$. Therefore the $\mathrm{Cs}^{+}$cations must be participating in the dynamic disorder, on a similar time scale to that of the $\mathrm{Pb}_{4}{ }^{4-}$ reorientations.

The d.c. electrical conductivity of $\mathrm{CsPb}$ rises sharply at the $\beta-\alpha$ transition, indicating a significant decrease in the gap in $\sigma(E)[6]$. It increases slowly with temperature in the $\alpha$ phase, and drops slightly at the melting point.

$\mathrm{Na}-\mathrm{Sn}$ is also observed to have a complex melting behavior, exhibited in the calorimetric data [7] shown in Fig. 3. The crystal structure at low temperature [8] is the same as in $\mathrm{CsPb}$ and the other Zintl compounds of $\mathrm{Sn}$ and $\mathrm{Pb}$ with the alkali metals (except $\mathrm{Li}$ ). To investigate, again, 
the possibility of dynamic disorder in the high-temperature solid phase, inelastic neutron scattering measurements were carried out at the IRIS and HET spectrometers at ISIS [9].

Quasielastic scattering spectra measured on the IRIS spectrometer again showed characteristic broadening in both the $\alpha$ solid and liquid phases. Especially remarkable, in view of the significant incoherent scattering cross section of $\mathrm{Na}$, was the absence of observable diffuse elastic scattering in the $\alpha$ phase, which was zero within experimental error when averaged over all angles except those near $Q=1 \AA^{-1}$, where there was significant Bragg scattering. The absence of significant incoherent elastic scattering indicates that the dynamic disorder associated with the $\mathrm{Na}^{+}$ ions is translational, rather than orientational, in nature. The intensities of the single lorentzian functions fitted to the spectra measured on IRIS and HET at each angle are shown as a function of $Q$ in Fig. 4. The HET results show a broad component whose width and intensity both increase considerably at higher $Q$; the dramatic rise in intensity above $1.5 \AA^{-1}$ is reminiscent of that found in $\mathrm{CsPb}$ (Fig. 2). This component is not seen in the IRIS data at higher $Q$, presumably because it is too broad to show up within the IRIS window ( $\pm 0.4 \mathrm{meV}$ ). On the other hand the IRIS data show a much narrower component whose intensity falls rapidly with $Q$ which is presumably too narrow to be picked out on top of the broad component in the poorer resolution HET data. It is reasonable to associate this narrow component with the $\mathrm{Na}^{+}$migration and the broad component with the more rapid reorientations of the $\mathrm{Sn}_{4}^{4-}$ polyanions.

Taken together; these results show that the dynamic disorder in the $\alpha$ phase of $\mathrm{NaSn}$ consists of rapid reorientations of the polyanions (fast rotor behavior) which enhance the slower cation migration (fast-ion conduction). This situation contrasts with that in $\mathrm{CsPb}$ where the polyanion rotations and cation motions appear to take place on the same time scale. Although the jump frequencies are different in $\mathrm{NaSn}$, the two processes must be strongly coupled since only one phase transition is observed prior to melting. 
The electrical transport in NaSn is coupled to the dynamic atomic disorder but in a different way from $\mathrm{CsPb}$. The electrical conductivity [10] shows a drop in conductivity at the $\beta-\alpha$ transition, presumably reflecting the additional scattering associated with the dynamic disorder, in contrast, any ionic component of the measured conductivity must increase at the transition. The large jump, by two orders of magnitude, observed in the conductivity of $\mathrm{CsPb}$ at the $\beta-\alpha$ transition is here found to take place within the $\alpha$ phase, reflecting a close coupling between atomic migration and electronic mobility [6]. There appears to be little if any change in the conductivity on melting.

\section{Phosphorus-Selenium}

The phosphorus-selenium system is best known for its glass-forming ability [11]: glasses can be readily formed by quenching from the melt over most of the compositional range, broken only in the region around the $\mathrm{P}_{4} \mathrm{Se}_{3}$ composition where the formation of a molecular crystal of that composition appears to preclude glass formation [12]. Crystalline $\mathrm{P}_{4} \mathrm{Se}_{3}$ exhibits two phase transitions in the solid as the temperature is increased. The orthorhombic $\alpha$ phase that is stable at room temperature transforms at $85^{\circ} \mathrm{C}$ to a rhombohedral $\beta$ phase that further transforms to a cubic $\gamma$ phase at $244^{\circ} \mathrm{C}$, before melting at $244^{\circ} \mathrm{C}$. On the basis of geometrical considerations, supported by diffraction data [13] and evidence from NMR [14], the $\beta$ and $\gamma$ phases are believed to be rotor phases with rapidly reorienting $\mathrm{P}_{4} \mathrm{Se}_{3}$ units. The two solid-solid transitions have considerable hysteresis, the $\gamma-\beta$ transition temperature being reduced by over $50^{\circ} \mathrm{C}$ on cooling from the melt; while the $\beta-\alpha$ is suppressed entirely and replaced by an orientational glass transition at around $-45^{\circ} \mathrm{C}$ $[13,15]$. According to the DSC analysis [15] shown in Fig. 5, the larger part of the entropy of melting, $11.3 \mathrm{~kJ} / \mathrm{mol}$, is taken up at the $\alpha-\beta$ transition. This is very close to the value of $R \ln 48$ $(11.5 \mathrm{~kJ} / \mathrm{mol}$ ) which would correspond to the configurational entropy associated with the 6 possible orientations of a trigonal molecule about the (nearly equivalent) body diagonal axes of the rhombohedral cell. The entropy changes at the $\beta-\gamma$ and melting transitions are much smaller -1.3 and $2.4 \mathrm{~kJ} / \mathrm{mol}$ respectively. 
To investigate the microscopic dynamics of the rotor phases, quasielastic scattering measurements were carried out on the IRIS spectrometer at temperatures of $20^{\circ} \mathrm{C}, 180^{\circ} \mathrm{C}$ and $200^{\circ} \mathrm{C}$, corresponding to the metastable quenched $\beta$ phase, stable $\beta$ phase just prior to the $\beta-\gamma$ transition, and the middle of the stable $\gamma$ phase. Details will be given in a later publication along with model fits to the data [16]. Quasielastic broadening was seen at all three temperatures, with the width increasing with temperature. Preliminary results for the intensities in the $\beta$ (averaging over the two temperatures) and $\gamma$ phases are shown in Fig. 6. Small but significant differences are seen between the two phases, indicating that the geometry of the molecular reorientations is sightly different in the two phases. If the $\beta$ phase is indeed characterized by reorientations between discrete orientations of the molecule in the rhombohedral cell, it is possible that the reorientations are isotropic in the cubic cell of the $\gamma$ phase. These suggestions will be tested by the model calculations referred to above.

\section{Conclusions}

Two alkali-metal — polyvalent metal Zintl compounds, $\mathrm{CsPb}$ and NaSn, exhibit a two-stage melting process with high-temperature solid phases characterized by rapid dynamical disorder. In $\mathrm{CsPb}$ this disorder is clearly associated with rapid reorientations of $\mathrm{Pb}_{4}^{4-}$ polyanions with the $\mathrm{Cs}^{+}$ cations participating in the dynamical disorder on the same time scale. In NaSn the disorder is associated with fast reorientations of the $\mathrm{Sn}_{4}{ }^{4-}$ polyanions closely coupled to a slower migration of the $\mathrm{Na}^{+}$cations. The two high-temperature solid phases of the molecular crystal $\mathrm{P}_{4} \mathrm{Se}_{3}$ are confirmed to be rotor phases with small but significant differences in the reorientational motions in the two phases.

\section{Acknowledgments}

The authors are grateful to J. E. Enderby, W. van der Lugt and H. Eckert for many illuminating discussions, K. J. Volin for preparation and characterization of the phosphorusselenium samples, and M. Adams and T. Perrin for helpful guidance with the IRIS and HET 
experiments on NaSn. They also acknowledge the invaluable support of the operations staffs at . IPNS, ILL and ISIS. This work was supported by the U.S. Department of Energy, Materials Sciences — Basic Energy Sciences, under Contract W-31-109-ENG-38. 


\section{References}

[1]. R. E. Marsh and D. P. Shoemaker, Acta Cryst. 6 (1953) 197.

[2]. H. T. J. Reijers, M.-L. Saboungi, D. L. Price, J. W. Richardson, K. J. Volin and W. van der Lugt, Phys. Rev. B 40 (1989) 6018.

[3]. B. P. Alblas, W. van der Lugt, J. Dijkstra, W. Geertsma and C. van Dijk, J. Phys. F 13 (1983) 2465; H. T. J. Reijers, M.-L. Saboungi, D. L. Price and W. van der Lugt, Phys. Rev. B 41 (1990) 5661.

[4]. G. K. Johnson and M.-L. Saboungi, J. Chem. Phys. 86 (1987) 6376; M.-L. Saboungi, H. T. J. Reijers, M. Blander and G. K. Johnson, J. Chem. Phys. 89 (1988) 5869.

[5]. D. L. Price, M.-L. Saboungi, H. T. J. Reijers, G. Kearley and R. White, Phys. Rev. Lett. 66 (1991) 1894; Phys. Rev. B 44 (1991) 7289.

[6]. J. Fortner, M.-L. Saboungi and J. E. Enderby, to be published.

[7]. M.-L. Saboungi, G. K. Johnson and D. L. Price, in Statics and Dynamics of Alloy Phase Transformations, Ed. P. E. A. Turchi and A. Gonis (Plenum, New York, 1994), p: 195.

[8]. W. Müller and K. Volk, Z. Naturforsch. 32B (1977) 709.

[9]. D. L. Price, M.-L. Saboungi and W. S. Howells, to be published.

[10]. M.-L. Saboungi, J. Fortner, W. S. Howells and D. L. Price, Nature 365 (1993) 237.

[11]. D. L. Price, M. Misawa, S. Susman, T. I. Morrison, G. K. Shenoy and M. Grimsditch, J. Non-Cryst. Solids 66 (1984) 443; M. Arai, R. W. Johnsion, D. L. Price, S. Susman, M: Gay and J. E. Enderby, J. Non-Cryst. Solids 83 (1986) 80.

[12]. E. Keulen and A. Vos, Acta Cryst. 12 (1959) 323.

[13]. R. Blachnik and U. Wickel, Thermochimica Acta 81 (1984) 185.

[14]. D. Lathrop and H. Eckert, J. Phys. Chem. 93 (1989) 7895.

[15]. J. R. Rollo, G. R. Burns, W. T. Robinson, R. J. H. Clark, H. M. Dawes and M. B. Hursthouse, Inorg. Chem. 29 (1990) 2889; J. R. Rollo and G. R. Burns, J. Non-Cryst, Solids 127 (1991) 242.

[16]. D. L. Price, M.-L. Saboungi, K. J. Volin and W. S. Howells, to be published. 


\section{Figure Captions}

Fig. 1. Enthalpy of $\mathrm{KPb}$ and $\mathrm{CsPb}$ as a function of temperature [4].

Fig. 2. Measured intensities (circles) of (a) lorentzian and (b) delta function peaks for $\mathrm{CsPb}$ at $888 \mathrm{~K}$, compared with those calculated from an orientational jump model (solid lines) [5].

Fig. 3. Enthalpy of NaSn as a function of temperature [7].

Fig. 4. Intensities of fitted lorentzian peaks for NaSn at $500^{\circ} \mathrm{C}: \bullet$ IRIS, o HET $(20$ $\mathrm{meV}), \triangle \mathrm{HET}(40 \mathrm{meV})$. The lines joining the points are a guide to the eye only.

Fig. 5. DSC results for $\mathrm{P}_{4} \mathrm{Se}_{3}$ [15]: first heating; --.-- subsequent heatings.

Fig. 6. Measured intensities of lorentzian peaks for $\mathrm{P}_{4} \mathrm{Se}_{3}$ in the $\beta$ (average of $20^{\circ} \mathrm{C}$ and $\left.180^{\circ} \mathrm{C}\right)$ and $\gamma\left(200^{\circ} \mathrm{C}\right)$ phases. 


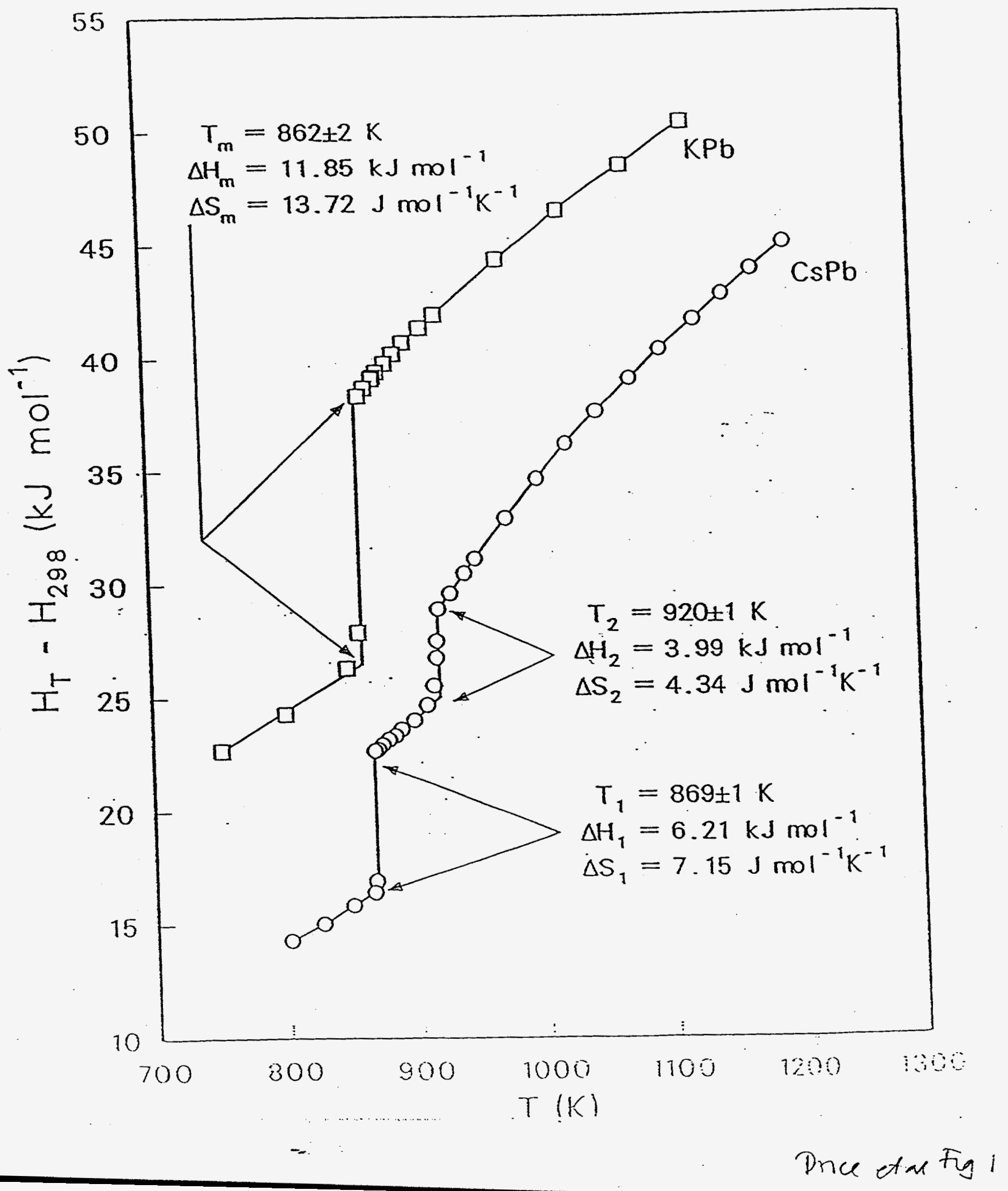



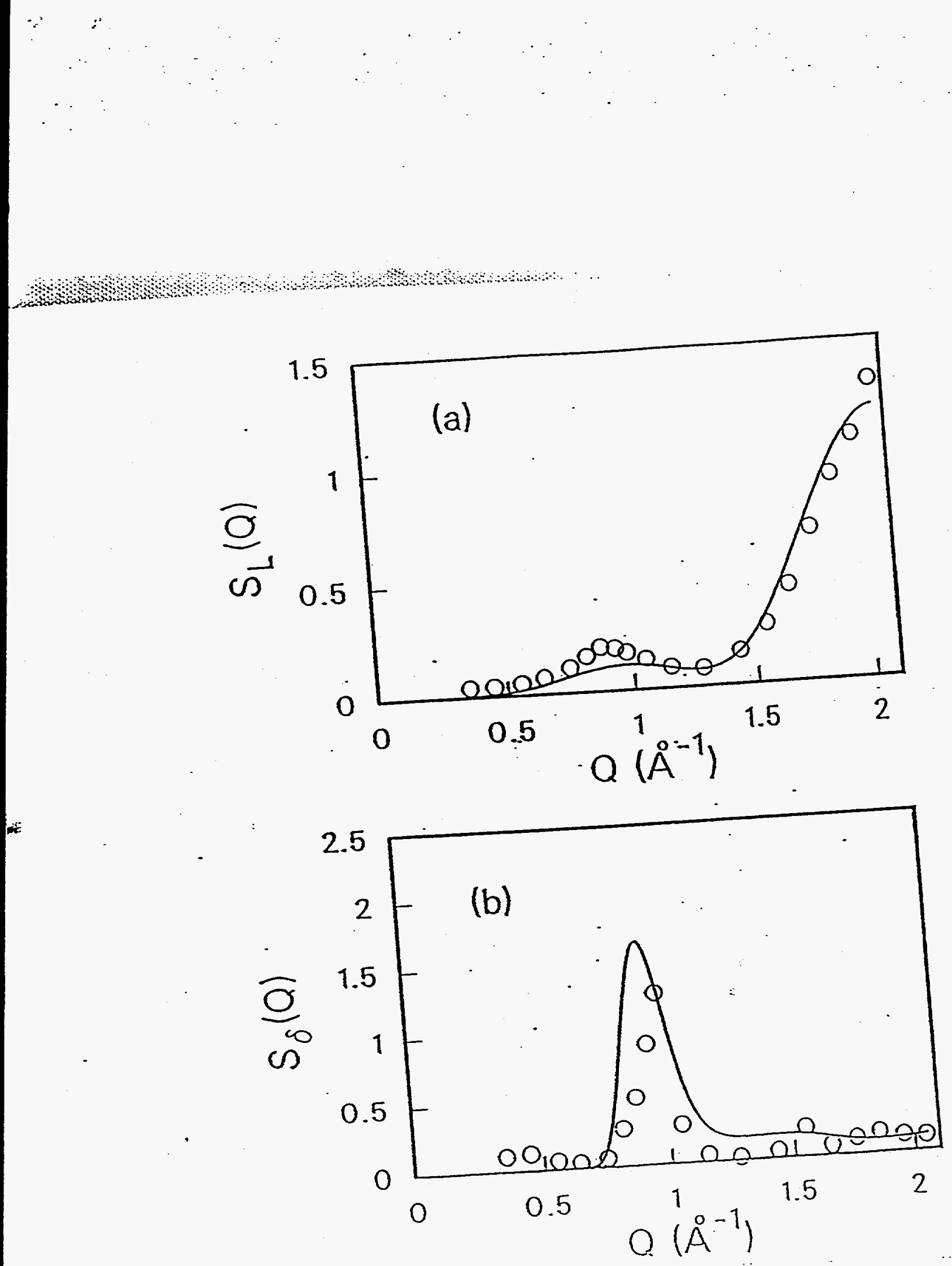

Pricedat Fog 


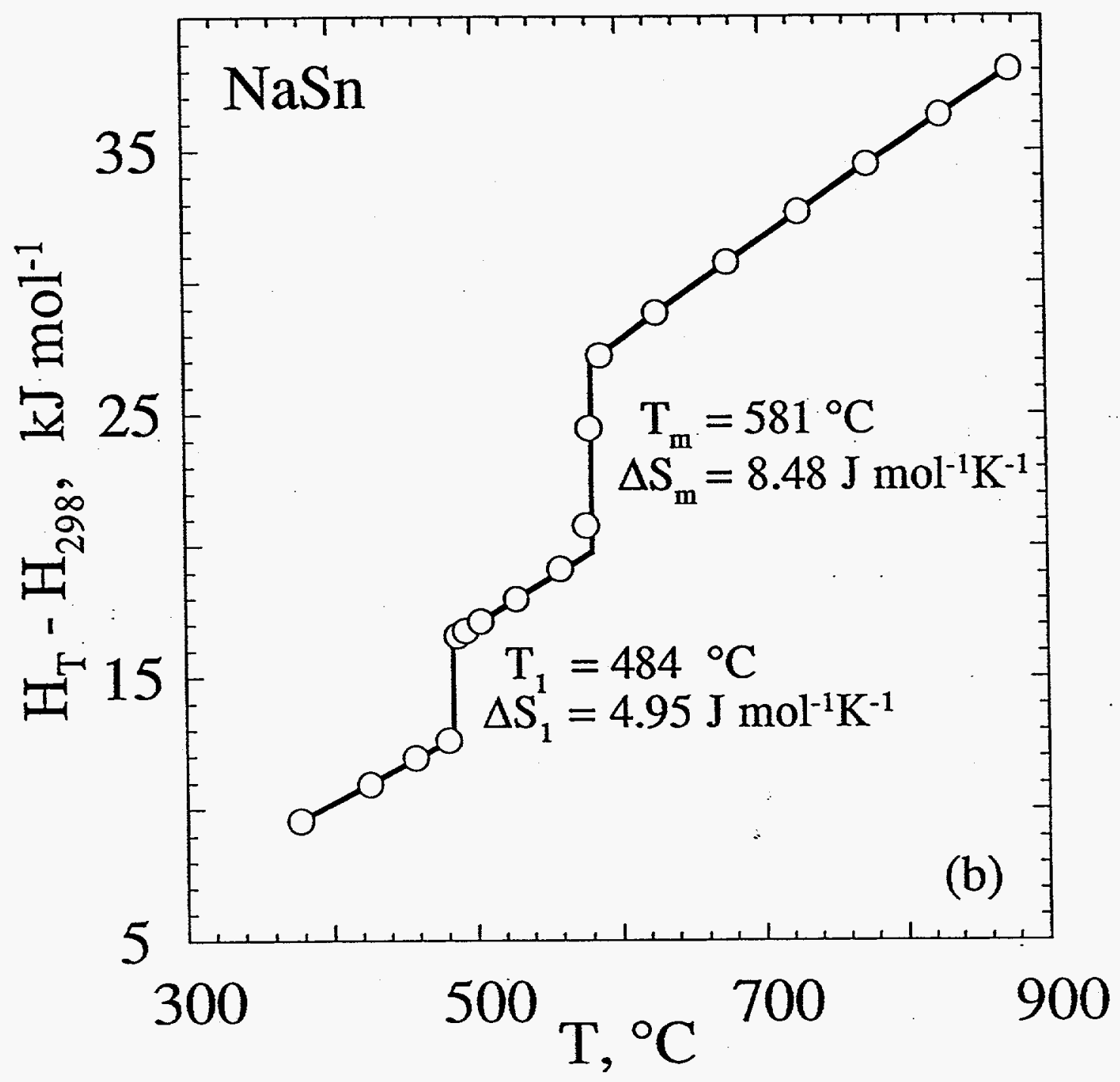

Prue etargis 
$S(Q)$

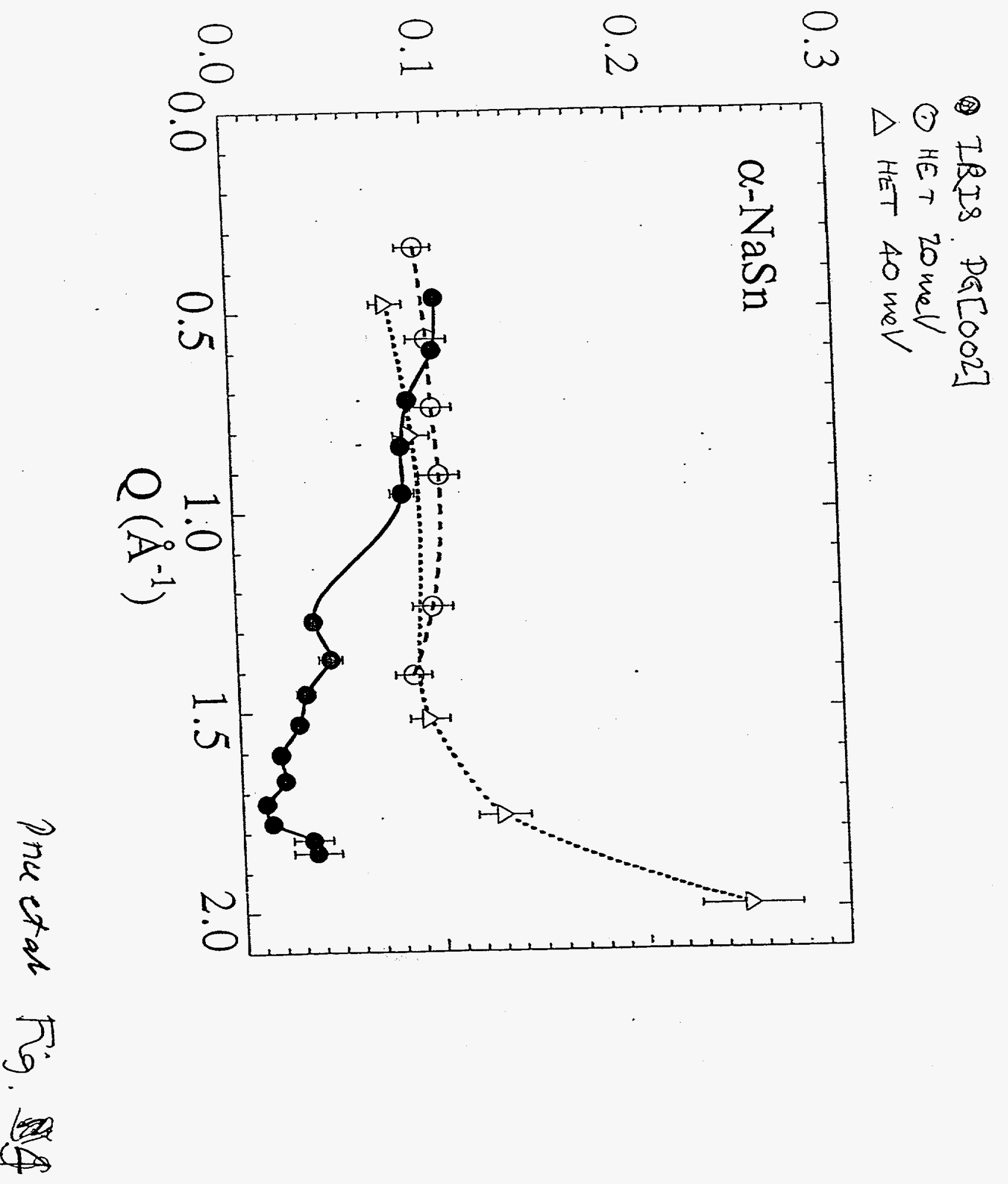




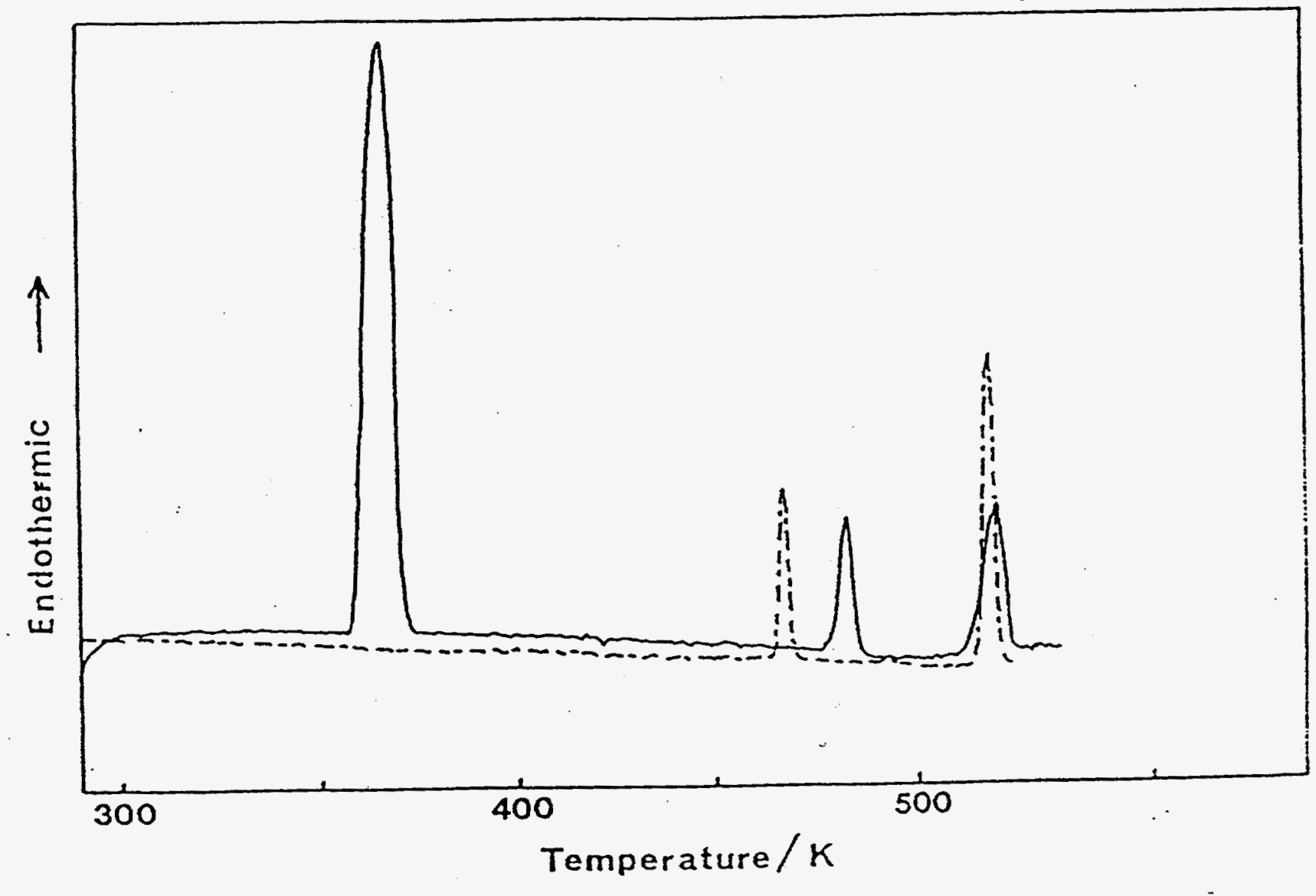

Pnue it at $\mathrm{Kg} 5$ 


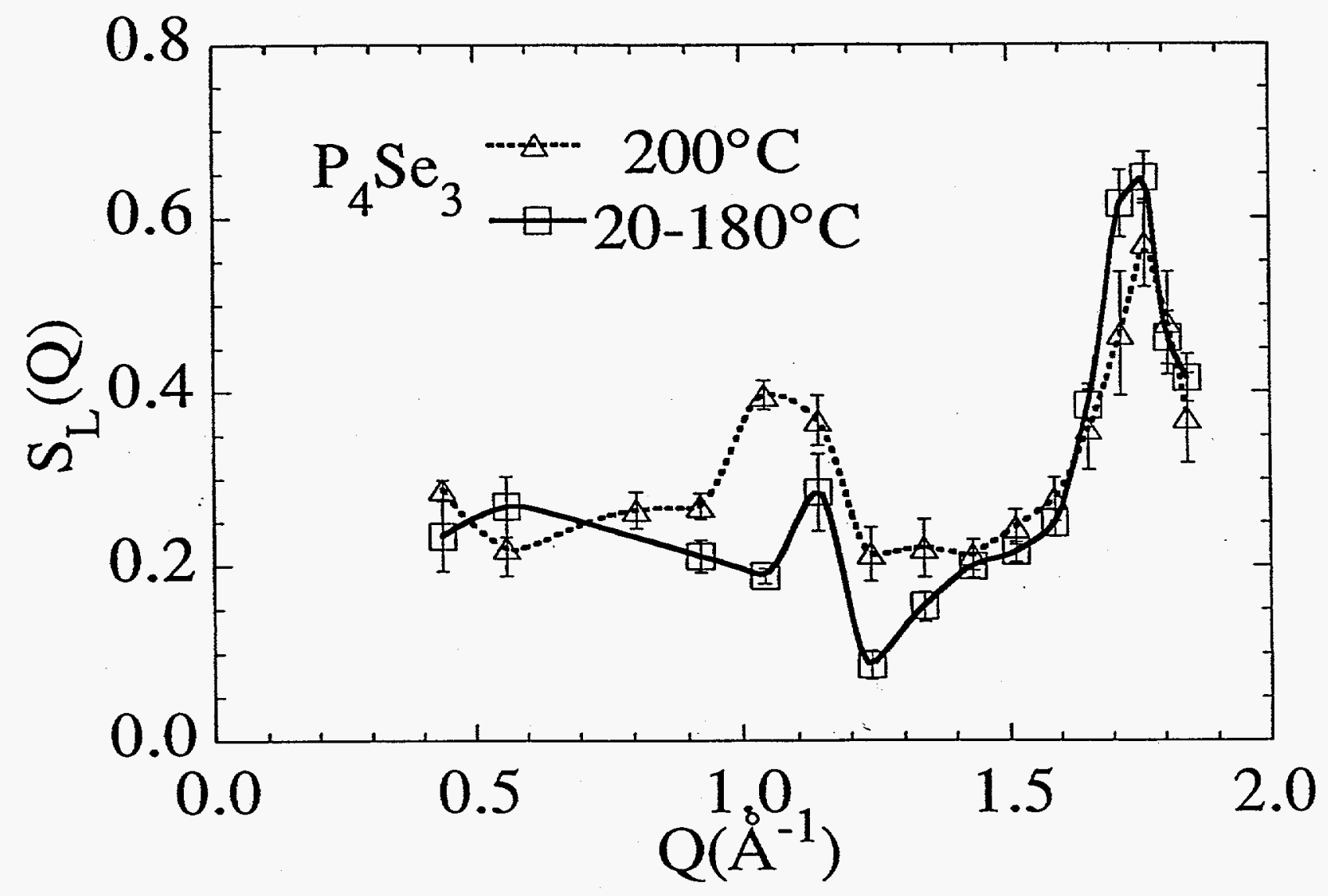

True the $\mathrm{Fg} \theta$ 